\title{
Suppression of Canine ATP Binding Cassette ABCB1 in Madin-Darby Canine Kidney Type II Cells Unmasks Human ABCG2-Mediated Efflux of Olaparib[
}

\author{
Yoo-Kyung Song, Ji Eun Park, Yunseok Oh, Sungwoo Hyung, Yoo-Seong Jeong, \\ Min-Soo Kim, Wooin Lee, and Suk-Jae Chung
}

College of Pharmacy, Seoul National University, Gwanak-gu, Seoul, Republic of Korea

Received April 27, 2018; accepted November 2, 2018

\begin{abstract}
Endogenous canine ATP binding cassette B1 (CABCB1) is expressed abundantly in Madin-Darby canine kidney type II (MDCKII) cells, and its presence often complicates phenotyping of the transport process. Errors in estimating the corrected efflux ratio (cER), as a result of the variable expression of $C A B C B 1$, were examined for the dual substrates of $A B C B 1$ and $A B C G 2$ in MDCKII cells expressing human ABCG2 (hABCG2). CABCB1 mRNA and protein expression was $60 \%$ and $55 \%$ lower, respectively, in MDCKII cells expressing hABCG2 compared with the wild type, suggesting that the expression of endogenous CABCB1 became variable after the expression of hABCG2. To minimize the contribution of endogenous efflux, CABCB1 was suppressed kinetically (using verapamil as a selective inhibitor) or biochemically
\end{abstract}

(transfecting short-hairpin RNA against CABCB1). Under these suppression conditions, $C E R$ values for irinotecan and topotecan (dual substrates of ABCB1 and ABCG2) were elevated by more than 4-fold and 2-fold, respectively, compared with cER values without the suppression. The cER of olaparib was similarly increased to 3- and 5-fold in MDCKII cells under the kinetic and biochemical suppression of CABCB1, respectively, suggesting that hABCG2-mediated efflux cannot be ruled out for olaparib. Since the substrate selectivity for ABCB1 and ABCG2 overlapped considerably, the possibility of an inaccurate estimation of CER must be considered for dual substrates in the case of the variable expression of cABCB1 in MDCKII cells.

\section{Introduction}

Madin-Darby canine kidney type II (MDCKII) cells are frequently used in bidirectional transport assays in studies of the functional involvement of efflux transporters (Tang et al., 2002). Reasons for the widespread use of this cell line may include a relatively rapid development in the tight junction, easy maintenance, and the fact that MDCKII cells expressing foreign transporters are readily available. In fact, some regulatory agencies such as the Food and Drug Administration (2017) recently recommended the use of MDCKII cells as one of the assay systems in phenotyping efflux transporters (e.g., ATP binding cassette ABCB1 and ABCG2) for substrates.

A portion of this work was previously presented as a poster presentation at the following meeting: Contribution of BCRP(ABCG2)-mediated transport of olaparib in MDCKII cells expressing BCRP and endogenous P-glycoprotein (2017) Song YK, Park JE, Oh Y, Hyung S, Jeong YS, Chung SJ 2017 American Association of Pharmaceutical Scientists Annual Meeting and Exposition; 2017 Nov 12-15; San Diego, CA.

This work was supported by the National Research Foundation of Korea [Grant 2009-0083533], funded by the Korean Ministry of Science, Information and Communications Technology, and Future Planning.

https://doi.org/10.1124/jpet.118.250225.

S This article has supplemental material available at jpet.aspetjournals.org.
Despite their utility, however, wild-type (WT) MDCKII cells express a relatively abundant number of canine transporters such as canine ABCB1 (cABCB1) (Goh et al., 2002), thereby complicating phenotyping of the transport process. The corrected efflux ratio (cER), which is the ratio of the efflux ratio (ER) calculated in cells expressing a foreign transporter to that in control cells, is frequently used to account for the contribution of endogenous transporters. In general, a cER value greater than 2 is regarded as a reasonable threshold (Food and Drug Administration, 2017) to indicate a contribution of an efflux transporter. This type of correction, however, would only be valid when the baseline function of endogenous transporters remains constant within the study period. In practice, however, the importance of demonstrating the stationary function of an endogenous transporter appears to be less well appreciated. For example, validation of the consistent function of endogenous transporters in cell lines is not mandatory in regulatory agency guidelines. In contrast, there is ample evidence in the literature demonstrating that routine maintenance can lead to the variable expression of endogenous transporters (Xia et al., 2005; Siissalo et al., 2007). In a recently reported study, Kuteykin-Teplyakov et al. (2010) showed that the functional expression of a foreign

ABBREVIATIONS: ABC, ATP binding cassette; cABC, canine ATP binding cassette; cER, corrected efflux ratio; DMSO, dimethylsulfoxide; ER, efflux ratio; GAPDH, glyceraldehyde 3-phosphate dehydrogenase; hABC, human ATP binding cassette; Ko143, (3S,6S,12aS)-1,2,3,4,6,7,12,12aoctahydro-9-methoxy-6-(2-methylpropyl)-1,4-dioxo-pyrazino[1',2':1,6]pyrido[3,4-b]indole-3-propanoic acid 1,1-dimethylethyl ester; MDCKII, Madin-Darby canine kidney type II; $\mathrm{P}_{\mathrm{app}}$, apparent permeability coefficient; PCR, polymerase chain reaction; qPCR, quantitative real-time polymerase chain reaction; shRNA, short-hairpin RNA; TPBS, Tween 20/phosphate-buffered saline; WT, wild type. 
transporter led to variations in the expression of endogenous transporters and thus to an inaccurate estimation of cER. It is noteworthy that the authors found that the functional expression of cABCB1 was reduced in MDCKII cells expressing human ABCB1 (hABCB1), resulting in a cER value of less than 1.5 (namely, the value indicating an unlikely substrate) for vinblastine, one of the most well characterized substrates of hABCB1 (Kuteykin-Teplyakov et al., 2010; Li et al., 2013). These observations suggest that the cER method, as it is currently used, is not reliable for phenotyping of the transport process in MDCKII cells when endogenous transporters are not expressed at constant levels.

In our routine transporter phenotyping study, we found that the basolateral to apical transport of olaparib (a known hABCB1 substrate) was strongly inhibited in MDCKII cells expressing hABCG2 in the presence of Ko143 [(3S,6S,12aS)$1,2,3,4,6,7,12,12 a$-octahydro-9-methoxy-6-(2-methylpropyl)1,4-dioxo-pyrazino $\left[1^{\prime}, 2^{\prime}: 1,6\right]$ pyrido[3,4-b]indole-3-propanoic acid 1,1-dimethylethyl ester], a standard hABCG2 inhibitor (Allen et al., 2002), at concentrations that would be ineffective in suppressing cABCB1 function. These results imply that the anticancer agent may be an hABCG2 substrate. However, this implication is in direct contradiction to the conclusions reported in a European Medicines Agency (2014) assessment for olaparib. In theory, such an inconsistency may be a manifestation of inadequate estimation of cER due to the variable function of an endogenous transporter such as cABCB1, although this possibility was not directly examined in the study cited. Therefore, the objectives of our study were to determine the possibility that endogenous cABCB1 is expressed at variable levels in MDCKII cells expressing hABCG2 and to assess its impact on estimating the cER of olaparib, a potential substrate for both ABCB1/ABCG2.

\section{Materials and Methods}

Chemicals and Reagents. $\left[{ }^{3} \mathrm{H}\right]$-Digoxin (specific activity, 39.8 $\mathrm{Ci} / \mathrm{mmol}$ ) and $\left[{ }^{3} \mathrm{H}\right]$-prazosin (specific activity, $84.2 \mathrm{Ci} / \mathrm{mmol}$ ) were purchased from PerkinElmer (Waltham, MA). Unlabeled digoxin, imipramine, Ko143 hydrate, verapamil, tariquidar, zosuquidar, and topotecan were obtained from Sigma-Aldrich (St. Louis, MO). Olaparib (purity greater than 99\%; LC Laboratories, Woburn, MA) was also used in this study. Unlabeled prazosin and irinotecan were purchased from Tokyo Chemical Industry (Tokyo, Japan) and Cayman Chemical (Ann Arbor, MI), respectively. High-performance liquid chromatography-grade methanol and formic acid were purchased from Thermo Fisher Scientific (Pittsburgh, PA) and Fluka (Cambridge, MA), respectively.

Cell Lines and Cultures. Cells from a WT MDCKII cell line (MDCKII/WT; passage numbers 47-60; European Collection of Authenticated Cell Culture, Salisbury, UK) and an LLC-PK1 cell line (passage numbers 196-221; American Type Culture Collection, Rockville, MD) were used in this study. MDCKII cells were grown in Dulbecco's modified Eagle's medium (Welgene Inc., Daegu, Korea) containing $10 \%$ fetal bovine serum (Welgene Inc.), $1 \%$ nonessential amino acid solution, $100 \mathrm{U} / \mathrm{ml}$ penicillin, and $0.1 \mathrm{mg} / \mathrm{ml}$ streptomycin under a humidified atmosphere of air containing $5 \% \mathrm{CO}_{2}$ at $37^{\circ} \mathrm{C}$ and were cultured under previously described conditions (Lee et al., 2015; Hyung et al., 2017; Yim et al., 2017).

Generation of MDCKII/hABCG2 and LLC-PK1/hABCG2 Cells. In this study, a commercially available plasmid construct containing cDNA for hABCG2 (pCMV6-AC; OriGene, Rockville, MD) was transfected to functionally express the human transporter.
MDCKII/WT cells (passage number 49) and WT LLC-PK1 cells (LLC-PK1/WT; passage number 202) were seeded in 12-well plates 24 hours prior to transfection. When the cells reached 50\%-70\% confluence, they were transfected using FuGENE HD Transfection Reagent (Promega, Madison, WI) according to the manufacturer's instructions. Briefly, $2 \mu \mathrm{g}$ plasmid DNA was transfected with $6 \mu \mathrm{l}$ transfection reagent mixed with Opti-MEM (Thermo Fisher Scientific), and the cells were transferred to a $100-\mathrm{mm}$ dish after 24 hours of transfection. Geneticin was introduced to the media at a concentration of 0.6 and $1 \mathrm{mg} / \mathrm{ml}$ for MDCKII and LLC-PK1 cells, respectively, on the next day of the cell transfer. The selection media were maintained for at least 14 days to select the clones resistant to the antibiotics. A Hoechst 33342 dye efflux assay (Scharenberg et al., 2002) was then carried out to select the clone having the most distinct functional expression (i.e., the least fluorescent clone) of the transporter. When necessary, semiquantitative polymerase chain reaction (PCR) analysis was carried out using the following the primers and cycling conditions: $5^{\prime}$-GCCTCACCTTATTGGCCTCA-3' and 5'-AGTTCCACGGCTGAAACACT-3' for $c A B C B 1,5^{\prime}$-AGATTGTCAGCAATGCCTCC-3' and 5'-GAGCTTGACAAAGTGGTCATT-3' for canine glyceraldehyde 3-phosphate dehydrogenase $(G A P D H)$, and 5'-GAGTGAACGGATTTGGCCGC-3' and 5' -TCTCATGGTTCACGCCCATC-3' for porcine GAPDH. PCR conditions consisted of denaturation at $94^{\circ} \mathrm{C}$ for 30 seconds, annealing at $56^{\circ} \mathrm{C}$ for 30 seconds, and elongation at $72^{\circ} \mathrm{C}$ for 2 minutes and 30 seconds. A bidirectional transport study of $\left[{ }^{3} \mathrm{H}\right]$-prazosin (primarily a substrate for hABCG2; along with unlabeled prazosin, final concentration of prazosin to be $1 \mu \mathrm{M}$ ) (Lepist et al., 2012; Food and Drug Administration, 2017) was carried out for further confirmation of the functional expression. In this study, the ER of prazosin at a value greater than 9 (for MDCKII/hABCG2, compared with the ER of 1 in MDCKII/WT) or 7 (for LLC-PK1/hABCG2, compared with the ER of 2.4 in LLC-PK1/WT) was accepted as an indication of the functional expression of the efflux transporter (Supplemental Figs. 1 and 2) and was used in subsequent studies.

In parallel, mock-transfected MDCKII and LLC-PK1 cells were also generated by transfecting the WT cells with the corresponding empty vector. For MDCKII cells, a bidirectional transport study of $\left[{ }^{3} \mathrm{H}\right]-$ digoxin (primarily a substrate for cABCB1; along with unlabeled digoxin, final concentration of digoxin to be $1 \mu \mathrm{M}$ ) was carried out to compare the functional activity of $\mathrm{cABCB} 1$ in transfected and untransfected cells. In our study, mock-transfected and MDCKII/WT cells showed comparable functional expression of cABCB1 (i.e., the ER of digoxin was 6.7 in MDCKII/mock cells, compared with an ER of 6.3 in MDCKII/WT cells). Thus, in subsequent studies, MDCKII/WT cells were considered to be functionally identical to MDCKII/mock cells and were used as controls in the estimation of cER.

Quantitative Real-Time PCR. To determine the extent of target mRNA in MDCKII cells, a quantitative real-time PCR (qPCR) study was carried out using standard protocols. Briefly, total RNA was extracted from MDCKII cells using the Hybrid-R RNA extraction kit (GeneAll, Seoul, Korea) and was reverse transcribed with an oligo dT primer using a PrimeScript RT-PCR kit (Takara, Shiga, Japan) according to the manufacturer's instructions. Amplification was detected by TOPreal qPCR $2 \times$ PreMIX with SYBR Green (Enzynomics, Daejeon, Korea) on a StepOnePlus Real-Time PCR System (Applied Biosystems, Foster City, CA). Thermocycling was carried out using a reaction mixture of $15 \mu \mathrm{l}$, which comprised $7.5 \mu \mathrm{l}$ premix, $0.1 \mu \mathrm{l}$ forward and reverse primers (final concentration at $0.1 \mu \mathrm{M}$ ), $6.3 \mu \mathrm{l}$ PCR-grade water, and $1 \mu \mathrm{l}$ target cDNA. Sequences for the forward and reverse primers, respectively, were 5'-TTGCTGGTTTTGATGATGGA-3' ${ }^{\prime}$ and 5'-CTGGACCCTGAATCTTTTGG-3' for $c A B C B 1$ (Kuteykin-Teplyakov et al., 2010) and 5'-ATTCCACGGCACAGTCAAG-3' and 5'-TACTCAGCACCAGCATCACC-3' for canine GAPDH (Kuteykin-Teplyakov et al., 2010; Gartzke et al., 2015). In this study, the reaction mixture that did not contain a template was considered to be a negative control. Thermocycling conditions consisted of an initial activation of DNA polymerase for 10 minutes at $95^{\circ} \mathrm{C}$, followed by 
50 cycles of amplification at $95^{\circ} \mathrm{C}$ for 10 seconds, $60^{\circ} \mathrm{C}$ for 15 seconds, and then $72^{\circ} \mathrm{C}$ for 30 seconds, with a final melting step of $95^{\circ} \mathrm{C}$ for 15 seconds. In this study, the level of canine GAPDH mRNA detected in the amplification was used for normalization of the level of the target mRNA.

Western Blot Analysis. Western blot analysis was carried out using total cell lysates. Briefly, MDCKII cells were lysed with lysis buffer containing protease inhibitors (Complete; Roche, Indianapolis, IN). After brief centrifugation $\left(10,000 \mathrm{~g}, 10\right.$ minutes, $\left.4^{\circ} \mathrm{C}\right)$, the resulting supernatant was analyzed with a bicinchoninic acid assay (Smith et al., 1985). Samples containing an equivalent amount of total protein $(60 \mu \mathrm{g})$ were mixed with Laemmli buffer (Laemmli, 1970) and heated at $50^{\circ} \mathrm{C}$ for 30 minutes. The heated samples were resolved by $7.5 \%$ SDS-PAGE. Subsequently, the blot was prepared using a poly(vinylidene) fluoride membrane and incubated in a $0.05 \%$ Tween 20/phosphate-buffered saline (TPBS) solution containing $3 \%$ bovine serum albumin for 1 hour to block nonspecific binding. For the detection of cABCB1, the membrane was probed with the commonly used anti-ABCB1 antibody C219 (dilution 1:200, 3\% bovine serum albumin in TPBS; Biolegend, San Diego, CA) (Kuteykin-Teplyakov et al., 2010; Gartzke and Fricker, 2014; Gartzke et al., 2015). As a gel-loading control, the membrane was probed using $\beta$-actin antibody (dilution 1:200, 3\% skim milk in TPBS; Cell Signaling Technology, Danvers, MA). After incubation with appropriate secondary antibodies conjugated with horseradish peroxidase (anti-mouse or anti-rabbit IgG horseradish peroxidase; Thermo Fisher Scientific), immunoreactive bands were visualized using enhanced chemiluminescence (Supersignal Femto and Pico reagents for the detection of $\mathrm{cABCB} 1$ and $\beta$-actin, respectively; Thermo Fisher Scientific) and an ImageQuant LAS4000 instrument (GE Healthcare, Piscataway, NJ).

Generation of Short-Hairpin RNA-Transfected MDCKII Cell Lines. To biochemically suppress the expression of endogenous cABCB1, plasmid constructs containing short-hairpin RNA (shRNA) against cABCB1 (cABCB1-shRNA; GenePharma, Shanghai, China) were custom designed and introduced to both MDCKII/WT and MDCKII/ hABCG2 cells. The transfection of cABCB1-shRNA was carried out with FuGENE HD Transfection Reagent (Promega) according to the manufacturer's instructions. Briefly, parental MDCKII cells were seeded in a 12-well plate 24 hours prior to transfection. Two micrograms of cABCB1-shRNA was added to a mixture consisting of $6 \mu \mathrm{l}$ transfection reagent and $100 \mu \mathrm{l}$ Opti-MEM, and the solution was added to MDCKII cells for transfection. The cells were then transferred to a $100-\mathrm{mm}$ dish after 24 hours of transfection. Hygromycin (the selection agent) was introduced the next day at a concentration of $0.2 \mathrm{mg} / \mathrm{ml}$. The selection media were maintained for at least 14 days to select the clones resistant to the antibiotics. The clones were further screened for their functional activity of cABCB1 by comparing the apparent permeability coefficient $\left(\mathrm{P}_{\mathrm{app}}\right)$ and ER of $\left[{ }^{3} \mathrm{H}\right]$-digoxin (primarily a substrate for cABCB1; along with unlabeled digoxin, the final total concentration of digoxin to be $1 \mu \mathrm{M}$ ) with bidirectional transport assays (Table 2). In addition, a qPCR study was carried out to confirm that the expression level of $c A B C B 1$ was reduced in the shRNA-transfected clones compared with that in nontransfected MDCKII/WT. In the clones showing a marked decrease in digoxin efflux (i.e., from an ER of approximately 7 to less than 1.3), cABCB1 mRNA levels were also reduced by $>70 \%$ compared with nontransfected cells (cABCB1 protein levels also decreased; Supplemental Fig. 3). Therefore, these clones were considered to have biochemically suppressed CABCB1 expression and were used in subsequent studies.

Evaluation of Representative hABCB1 Inhibitors for Their Inhibitory Potency toward cABCB1 in MDCKII Cells. For evaluation of the inhibitory potency of representative ABCB1 inhibitors (e.g., tariquidar, cyclosporine A, zosuquidar, and verapamil; Stephens et al., 2001; Taub et al., 2005; Agarwal et al., 2010; Römermann et al., 2015) toward cABCB1, the basolateral to apical or apical to basolateral transport of $\left[{ }^{3} \mathrm{H}\right]$-digoxin (primarily a substrate for cABCB1; along with unlabeled digoxin, with the final total concentration of digoxin being $1 \mu \mathrm{M}$ ) was determined in MDCKII/WT cells in the presence of various concentrations of inhibitors. In addition, the transport of $\left[{ }^{3} \mathrm{H}\right]$ prazosin (primarily a substrate for hABCG2; along with unlabeled prazosin, the final total concentration of prazosin being $1 \mu \mathrm{M}$ ) was examined in MDCKII/hABCG2 cells for evaluation of the inhibitory potency of the inhibitors toward hABCG2.

Bidirectional transport studies were carried out as described previously (Jeong et al., 2017). Briefly, MDCKII cells were seeded on Transwell filters (12 $\mathrm{mm}$ diameter, $0.4 \mu \mathrm{m}$ pore size; Corning, Corning, NY) at a density of $0.5 \times 10^{6} \mathrm{cells} / \mathrm{ml}$ and then cultured for 5 to 6 days before being used in transport assays. Transport was initiated by adding transport buffer containing the substrate (i.e., digoxin or prazosin) to the donor compartment, followed by incubation at $37^{\circ} \mathrm{C}$ for 120 minutes. Aliquots $(300 \mu \mathrm{l})$ of the receiver and donor samples were collected at the end of the incubation. Scintillation fluid (Ultima Gold; PerkinElmer) was subsequently added to the samples and radioactivity was determined by liquid scintillation counting (Tri-Carb 3110 TR; PerkinElmer). In this study, the final concentration of dimethylsulfoxide (DMSO) (an agent used for the solubilization of $\mathrm{ABCB} 1$ inhibitors) in the transport buffer was equal to or below $0.1 \%$ in all inhibition experiments except for cyclosporine A (i.e., the final concentration of DMSO was $0.5 \%$ ). From our preliminary experiment, DMSO at a concentration below $1 \%$ in the transport medium was found to have no appreciable impact on transporter function, consistent with other literature findings (Taub et al., 2002).

Transcellular Transport of Dual Substrates of ABCB1 and ABCG2. The transcellular transport of dual substrates of ABCB1 and ABCG2 (e.g., irinotecan and topotecan; final concentration of $10 \mu \mathrm{M}$ ) was determined in the presence of $100 \mu \mathrm{M}$ verapamil in MDCKII/WT and MDCKII/hABCG2 cells (subsequently referred to as verapamiltreated cells) or in MDCKII/WT and MDCKII/hABCG2 cells expressing cABCB1-shRNA (subsequently referred to as shRNA-transfected cells). Olaparib was also included (at a final concentration of $10 \mu \mathrm{M}$ ) in this transport assay, since this drug could potentially serve as a substrate for both transporters. Transport of the dual substrates was studied for a period of 120 minutes at $37^{\circ} \mathrm{C}$. When necessary, $1 \mu \mathrm{M}$ Ko143 (a standard inhibitor of hABCG2; Supplemental Fig. 4) was added to the transport media to further confirm hABCG2-mediated transport. For chromatographic quantification of the dual substrates, previously reported assays were used with minor modifications (Luo et al., 2002; de Vries et al., 2007; Sparidans et al., 2011). In this study, the system was equipped with a Waters e2695 high-performance liquid chromatography system (Milford, MA) and an API 3200 QTRAP mass spectrometer (Applied Biosystems). Separations were carried out using a linear gradient of $0.1 \%$ formic acid in methanol and $0.1 \%$ formic acid in water at a flow rate of $0.25 \mathrm{ml} / \mathrm{min}$ with a reversed-phase high-performance liquid chromatography column (Eclipse XDB-C18, particle size $3.5 \mu \mathrm{m}$, internal diameter and length of the column $2.1 \times$ $100 \mathrm{~mm}$; Agilent, Santa Clara, CA). Samples were monitored at the following Q1/Q3 transitions (mass-to-charge ratio): 587.2/124.1 for irinotecan, 422.9/378.0 for topotecan, 435.2/281.0 for olaparib, and 281.3/86.1 for imipramine (an internal standard of the assay). The specificity, linearity, precision, and accuracy of the assay were within the acceptance criteria of assay validation guidelines (Food and Drug Administration, 2013). The limit of quantification was 10, 20, and $10 \mathrm{nM}$ for irinotecan, topotecan, and olaparib, respectively.

Transcellular Transport of Olaparib in LLC-PK1 Cells. The transcellular transport of olaparib was also determined in hABCG2overexpressing LLC-PK1 cells. Cells were seeded onto Transwell filters (12 mm diameter, $0.4 \mu \mathrm{m}$ pore size; Corning) at a density of $0.5 \times 10^{6}$ cells $/ \mathrm{ml}$ and were cultured for 5 to 6 days prior to the transport assays. Transport was initiated by adding transport buffer containing $10 \mu \mathrm{M}$ olaparib to the donor compartment, followed by incubation at $37^{\circ} \mathrm{C}$ for 120 minutes. When necessary, $1 \mu \mathrm{M}$ Ko143 (a standard inhibitor of hABCG2) was included in the transport media. Aliquots of the donor and receiver samples were collected at the end of the incubation and were subjected to chromatographic quantification as described above. 
Data Analysis. When it was necessary to estimate the apparent permeability coefficient ( $\mathrm{P}_{\text {app }}$ ), the following equation was used (eq. 1):

$$
\mathrm{P}_{\mathrm{app}}=\frac{1}{A} \times \frac{1}{C_{0}} \times \frac{d Q}{d t}
$$

where $d Q / d t, A$, and $C_{0}$ represent the transport rate, the surface area of the insert, and the initial concentration of the compound in the donor compartment, respectively. The ER was calculated by dividing the basolateral to apical $\mathrm{P}_{\text {app }}$ by the apical to basolateral $\mathrm{P}_{\text {app }}$ (Wang et al., 2005). In inhibition studies, the percent ER was also calculated by dividing the value for the ER in the presence of the inhibitor by that without the inhibitor (i.e., control). When necessary, the percent ER value was used to evaluate the half maximal inhibitory concentration $\left(\mathrm{IC}_{50}\right)$ by a nonlinear regression analysis using WinNonlin Professional 5.0.1 software (Pharsight Corporation, Mountain View, CA) and the following equation (eq. 2):

$$
V=V_{\max }-\left(V_{\max }-V_{0}\right) \times\left[\frac{[I]}{[I]+\mathrm{IC}_{50}}\right]
$$

where $V, V_{\max }, V_{0}$, and $[I]$ are the rate of transport in the presence of inhibitor, the maximal rate of transport, the basal rate of transport, and the concentration of inhibitor, respectively. Assuming the mechanism of inhibition to be competitive, the inhibitory constant $\left(K_{\mathrm{i}}\right)$ of the inhibitor was calculated for the estimated $\mathrm{IC}_{50}$ by using the following equation (eq. 3) (Cheng and Prusoff, 1973):

$$
K_{\mathrm{i}}=\frac{\mathrm{IC}_{50}}{1+\frac{[S]}{K_{\mathrm{m}}}}
$$

where $[S]$ is concentration of the substrate and $K_{\mathrm{m}}$ represents the Michaelis-Menten constant. For comparison of the apparent selectivity of cABCB1 inhibitors, the ratio of $K_{\mathrm{i}}$ values, in which $K_{\mathrm{i}, \mathrm{hABCG}}$ (estimated $K_{\mathrm{i}}$ with MDCKII/hABCG2) is divided by $K_{\mathrm{i}, \mathrm{ABCB} 1}$ (estimated $K_{\mathrm{i}}$ with MDCKII/WT), was calculated for the inhibitors.

When necessary, the cER was calculated by dividing the ER obtained in MDCKII/hABCG2 by the ER obtained in MDCKII/WT (eq. 4):

$$
\mathrm{cER}=\frac{\mathrm{ER}_{\mathrm{MDCKII} / \mathrm{hABCG} 2}}{\mathrm{ER}_{\mathrm{MDCKII} / \mathrm{WT}}}
$$

Statistical Analysis. For comparison of means between or among groups, the two-tailed/unpaired $t$ test (for qPCR studies) or the oneway analysis of variance (for bidirectional transport studies), followed by the Tukey post hoc test, was used. In this study, a value of $P<0.05$ was considered statistically significant.

\section{Results}

Variable Expression of cABCB1 in MDCKII Cell Lines. We compared the endogenous expression of cABCB1 between MDCKII/WT and MDCKII/hABCG2 cells. The qPCR results indicated that the mRNA levels of $c A B C B 1$ were approximately $60 \%$ lower in MDCKII/hABCG2 than in MDCKII/WT cells (Fig. 1A; $n=3$ independently prepared sets). The mRNA levels of $c A B C G 2$ were quite low, with threshold cycles greater than 33 in both MDCKII/WT and MDCKII/hABCG2 cells, which is consistent with a literature report indicating that $c A B C G 2$ is not abundantly expressed in MDCKII cells (data not shown) (Di et al., 2011). The observed downregulation of $c A B C B 1$ mRNA was accompanied by a decrease in the cABCB1 protein level. Multiple immunoreactive bands were detected when the immunoblotting analysis was performed with the commonly used ABCB1 antibody C219, which can react with cABCB1 (Ito et al., 1999; Kuteykin-Teplyakov et al., 2010; Römermann et al., 2015). The band near $130 \mathrm{kDa}$ likely comes from crossreactivity with ABCB4, according to previous reports (Schinkel et al., 1991; Scheffer et al., 2000). Thus, densitometric analysis was performed on the two upper bands with electrophoretic mobility of $150-170 \mathrm{kDa}$, likely arising from differing degrees of post-translational modifications including glycosylation, as reported previously (Tang et al., 2002). When the band intensities were compared using three independently prepared sets of samples, MDCKII/hABCG2 cells displayed lower cABCB1 protein levels than MDCKII/WT cells (on average by $55 \%$; Fig. 1B). Among the independently prepared samples (passage number differences within 5), cABCB1 protein levels also varied. Intriguingly, the cABCB1 level was consistently lower in MDCKII/hABCG2 cells than in MDCKII/WT cells. These collective observations indicate that cABCB1 expression in MDCKII cells was reduced in MDCKII/hABCG2 compared with MDCKII/WT cells. We reasoned that the downregulation of cABCB1 may lead to a violation of the assumption regarding the consistency of
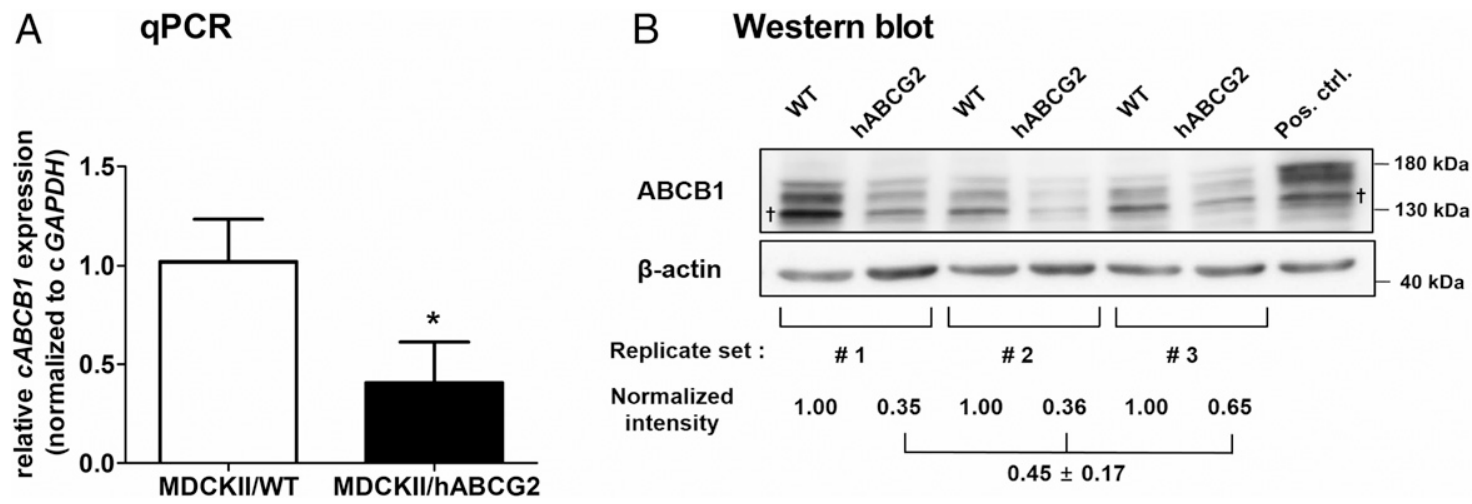

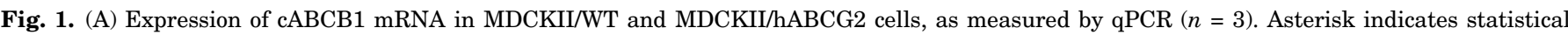

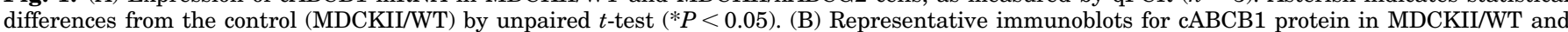

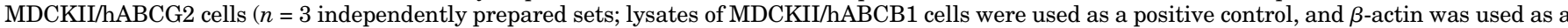

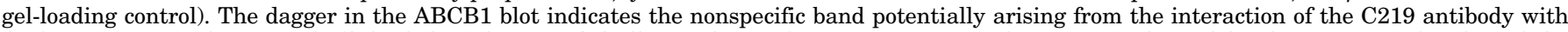

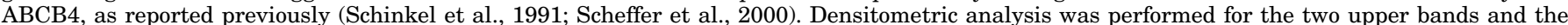
relative intensities were obtained by comparing the signals of the samples within the same set. Data are expressed as the mean \pm S.D. 
baseline transport, thereby negatively affecting the accuracy of the cER, especially for dual substrates of cABCB1 and hABCG2.

Differential Inhibition of cABCB1 and hABCG2 in MDCKII/hABCG2 Cells in the Presence of hABCB1 Inhibitors. In this study, the comparative selectivity of commercially available hABCB1 inhibitors (tariquidar, cyclosporine A, zosuquidar, and verapamil; Stephens et al., 2001; Taub et al., 2005; Agarwal et al., 2010; Römermann et al., 2015) toward cABCB1 and hABCG2 was examined in MDCKII/WT and MDCKII/hABCG2 cells. We measured the apical to basolateral and basolateral to apical transport of digoxin (an index for cABCB1 function) in the presence of various levels of inhibitors and calculated the $\mathrm{IC}_{50} / K_{\mathrm{i}}$ values (Fig. 2; Table 1). Tariquidar was an inadequate inhibitor of cABCB1 at the concentration reported to inhibit ABCB1 (i.e., $0.2 \mu \mathrm{M}$ ) (Römermann et al., 2015). Furthermore, at higher concentrations, tariquidar and cyclosporine A were significant inhibitors of both cABCB1 and hABCG2: $K_{\mathrm{i}}$ values toward hABCG2 and cABCB1 were not substantially different between each other (i.e., fold differences of less than 10). hABCG2 was not inhibited by any of the zosuquidar concentrations tested; however, this inhibitor had limited solubility in the aqueous media of the transport study, and it was not
A
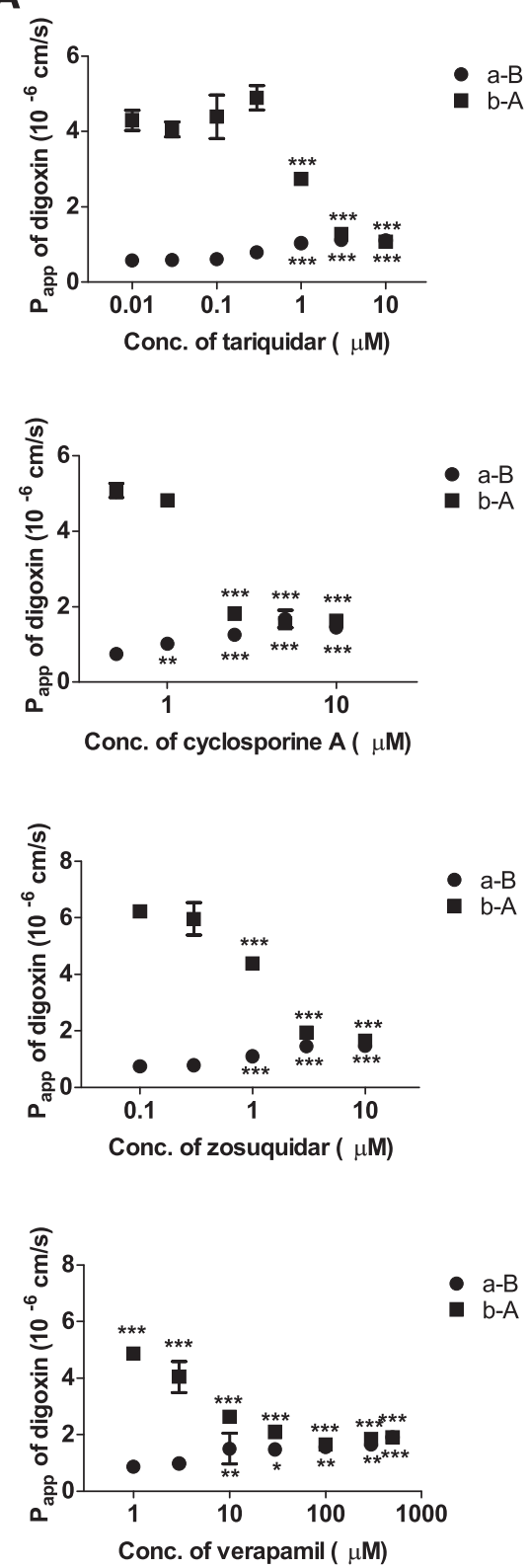

B
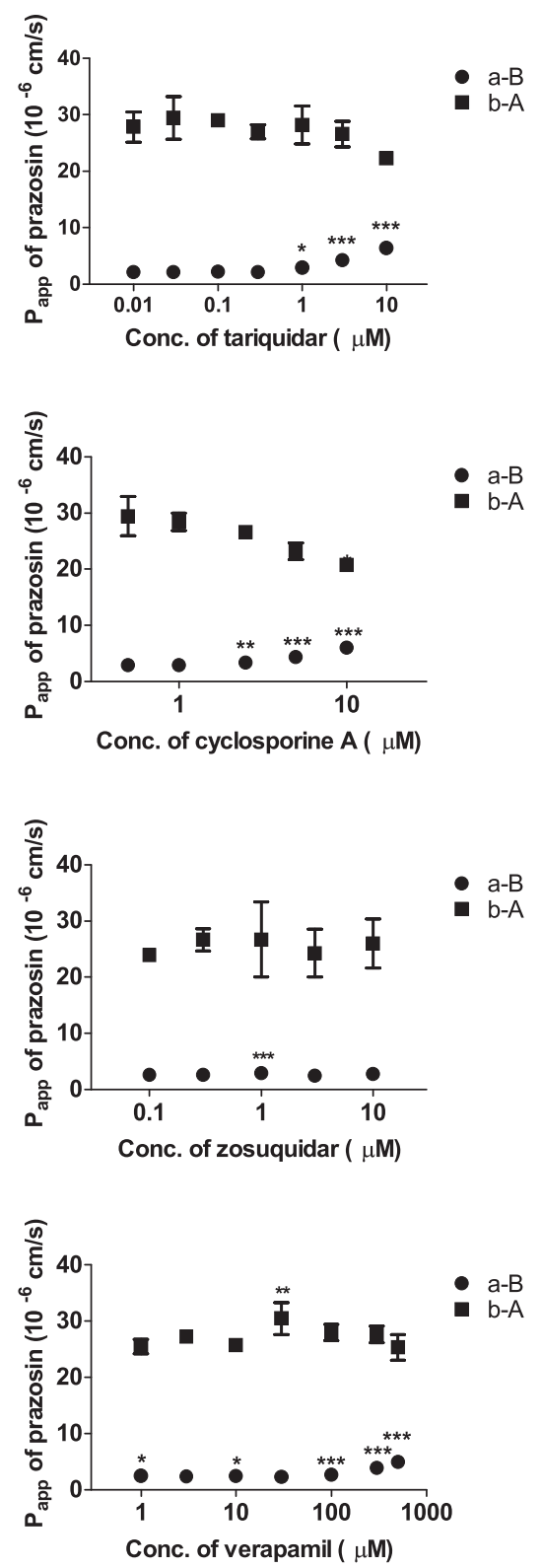

C

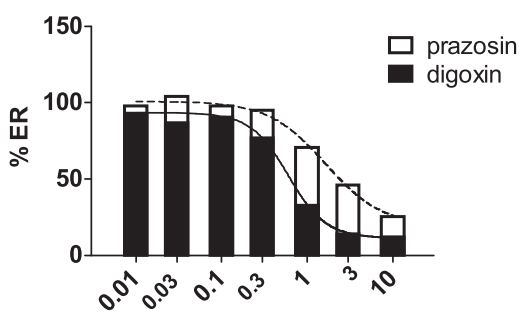

Conc. of tariquidar $(\mu \mathrm{M})$
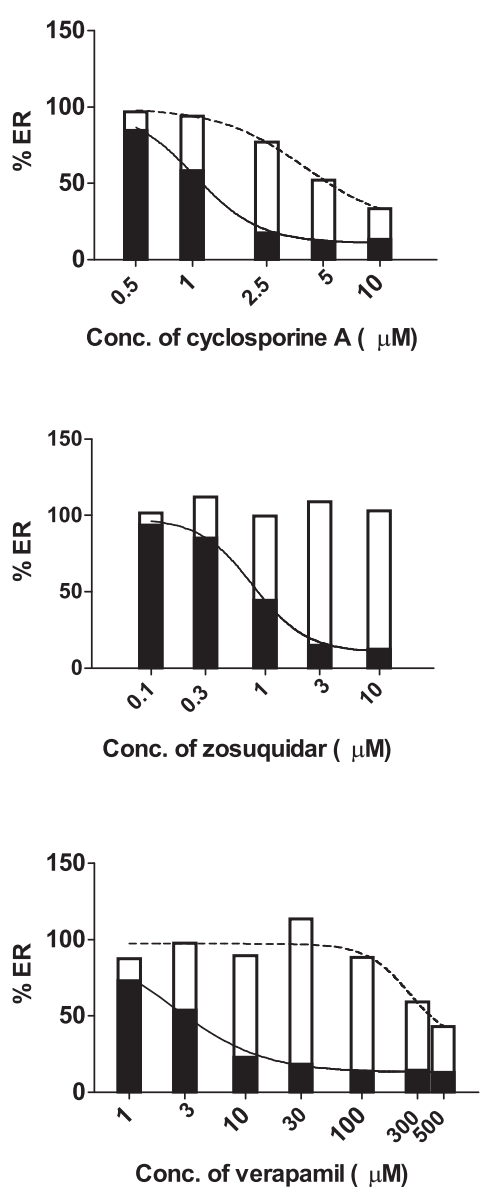

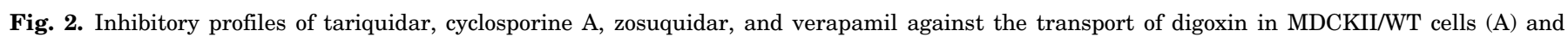

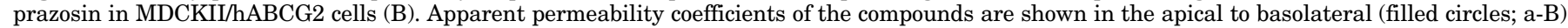

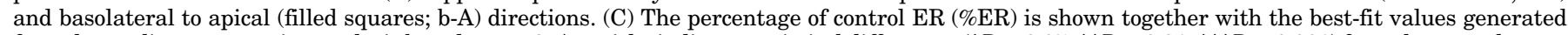

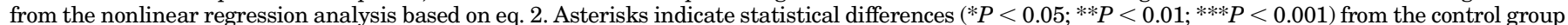

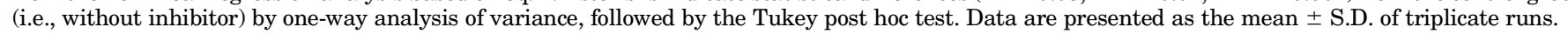


TABLE 1

$\mathrm{IC}_{50}$ and $K_{\mathrm{i}}$ values of some representative ABCB1 inhibitors

\begin{tabular}{|c|c|c|c|c|c|}
\hline \multirow{2}{*}{ Inhibitor } & \multicolumn{2}{|c|}{ hABCG2 } & \multicolumn{2}{|c|}{ cABCB1 } & \multirow{2}{*}{$\begin{array}{c}K_{\mathrm{i}} \text { Ratio } \\
\left(K_{\mathrm{i}, \mathrm{ABCG} 2} / K_{\mathrm{i}, \mathrm{CABCB} 1}\right)\end{array}$} \\
\hline & $\mathrm{IC}_{50}$ & $K_{\mathrm{i}}$ & $\mathrm{IC}_{50}$ & $K_{\mathrm{i}}$ & \\
\hline \multicolumn{6}{|c|}{$\mu M$} \\
\hline Tariquidar & 2.19 & 2.17 & 0.491 & 0.482 & 4.51 \\
\hline Cyclosporine A & 4.69 & 4.65 & 0.788 & 0.774 & 6.01 \\
\hline Zosuquidar & $>10$ & $>9.8$ & 0.617 & 0.606 & $>16.2$ \\
\hline Verapamil & 417 & 414 & 2.21 & 2.17 & 191 \\
\hline
\end{tabular}

$K_{\mathrm{i}}$ values for hABCG2 and cABCB1 were calculated by using the $K_{\mathrm{m}}$ value of prazosin toward hABCG2 (i.e., $128 \mu \mathrm{M}$ ) and the $K_{\mathrm{m}}$ value of digoxin toward cABCB1 (i.e., $53.9 \mu \mathrm{M}$ ), estimated from the $\mathrm{P}_{\text {app }}$ from the apical to basolateral side in MDCKII/hABCG2 and MDCKII/WT cells, respectively.

possible to increase its concentration further. Therefore, based on the kinetic estimates of $K_{\mathrm{i}}$, the difference in $K_{\mathrm{i}}$ values for the transporters was calculated to be approximately 16.2 for zosuquidar. Among the inhibitors studied, verapamil showed relatively higher selectivity for inhibition toward cABCB1 ( $K_{\mathrm{i}}$ ratio of 191 , indicating a 191-fold lower $K_{\mathrm{i}}$ value toward cABCB1). In subsequent studies, the presence of $100 \mu \mathrm{M}$ verapamil (Figs. 3 and 4) was assumed to have functional inhibition toward cABCB1 with no appreciable inhibition toward hABCG2. In addition, our sensitivity analysis indicated that $100 \mu \mathrm{M}$ verapamil would permit adequate selectivity to be maintained in a wide range of substrates having $K_{\mathrm{m}}$ values from 1 to $100 \mu \mathrm{M}$ (Supplemental Fig. 5).

Transport of Dual Substrates in the Presence of Functional Inhibition/Reduced Expression of cABCB1 in MDCKII Cells. To determine whether suppressing the function and expression of cABCB1 would result in improved estimation of the $\mathrm{cER}$, control studies were carried out using MDCKII cells in the presence of $100 \mu \mathrm{M}$ verapamil (i.e., verapamil-treated) and in MDCKII cells expressing shRNA directed to cABCB1 (i.e., shRNA-transfected) for the dual substrates of ABCB1 and ABCG2. In this study, the ER values of digoxin (primarily a substrate for cABCB1) were reduced to close to unity in MDCKII cells under the two suppressive conditions (i.e., theoretically, an adequate suppression of cABCB1 activity/expression would result in an ER of 1) (Fig. 3A; Table 2). In contrast, the cER of prazosin (primarily a substrate for hABCG2) in the two conditions under which cABCB1 functions were suppressed appeared to be unaltered (i.e., the value remained in the range of 9.9-12.5; Fig. 3B; Table 2; as a reference, the value for $\mathrm{cER}$ without suppression was 9.6), which is indicative of no meaningful reduction in hABCG2 function under conditions where cABCB1 function was suppressed. As expected, the ER values for olaparib, irinotecan, and topotecan (i.e., dual substrates) in MDCKII/WT cells were high without suppression, whereas the ER values were reduced to close to unity when cABCB1 function was inhibited either kinetically or biochemically (Fig. 3A; Table 2) in MDCKII/WT cells. As a result, the cER values of irinotecan and topotecan were elevated by 4.8-fold and 2.3-fold for verapamil-treated cells and 4.5-fold and 2.4-fold for shRNA-transfected cells, respectively (Fig. 3B; Table 2). Collectively, the above observations suggest that the two suppressive methods are both effective in improving cER estimation for dual substrates of ABCB1/ABCG2 in MDCKII cells expressing hABCG2.

Estimation of the cER for Olaparib in MDCKII Cells Expressing hABCG2 under the Functional Suppression of CABCB1. In this study, we found that the ER values for olaparib in MDCKII/hABCG2 and MDCKII/WT cells were comparable (Fig. 4A; namely, cER estimation close to 1 ), which suggests that olaparib is not a substrate for hABCG2 and is consistent with previously published observations (McCormick and Swaisland, 2016). In contrast, the ER for olaparib in MDCKII/hABCG2 cells was significantly decreased in the presence of Ko143 (Fig. 4A). Under verapamil treatment conditions, the ER in MDCKII/WT cells was markedly reduced but the extent of the reduction was less in MDCKII/hABCG2 cells. Similarly, the ER for olaparib was markedly decreased in shRNA-transfected MDCKII/WT cells. As a result, the cER of olaparib was $2.81 \pm 0.376$ (verapamil treatment, $P<0.01$ ) or $4.62 \pm 0.421$ (shRNA transfection, $P<0.01$ ) compared with 0.926 \pm 0.0889 (without cABCB1 suppression). The addition of Ko143 resulted in a further reduction in the $\mathrm{ER}(P<0.01$; Fig. $4, \mathrm{~B}$ and $C)$. Furthermore, the addition of elacridar and fumitremorgin $C$ also resulted in a reduction of the ER to 0.97-1.20 (Supplemental Fig. 6). Collectively, these observations indicate that the possibility of hABCG2-mediated transport cannot be excluded for olaparib.

hABCG2-Mediated Transport of Olaparib in hABCG2Overexpressing LLC-PK1 Cells. When transcellular transport of olaparib was determined in hABCG2-overexpressing LLC-PK1 cells (LLC-PK1/hABCG2), the ER was significantly elevated for olaparib compared with LLC-PK1/WT or LLCPK1/mock cells, resulting in a cER of $1.88 \pm 0.192$ or $2.20 \pm$ 0.571 , respectively. Upon the addition of Ko143, the ER value was reduced comparably to control cells (Fig. 5).

\section{Discussion}

The findings reported in this study indicate that cABCB1 expression was significantly reduced in MDCKII cells expressing
A
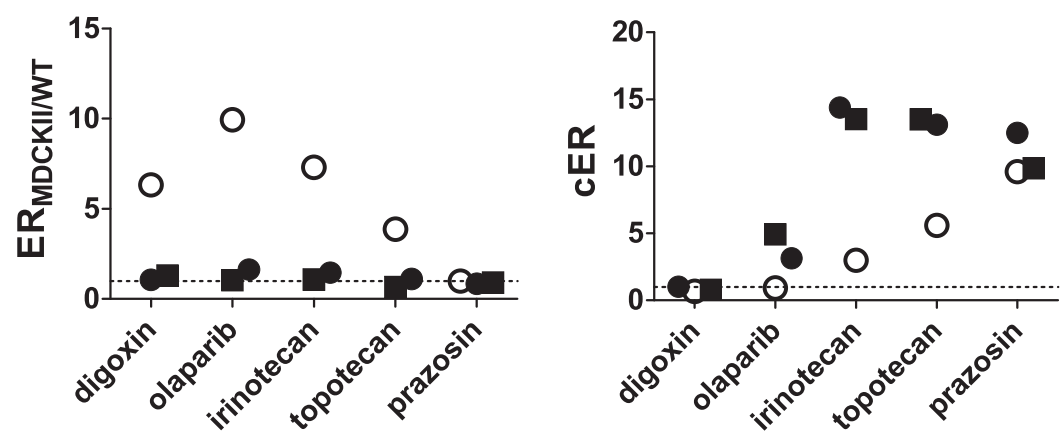

Fig. 3. (A) Estimate of ER values for $\mathrm{ABCB} 1$ and/or $\mathrm{ABCG} 2$ substrates in MDCKII/WT cells. (B) Estimate of cER values for ABCB1 and/or ABCG2 substrates, calculated from ER values obtained for MDCKII/WT and MDCKII/hABCG2 cells. Open circles indicate no suppression, whereas filled circles and filled squares indicate the verapamil-treated and shRNA-transfected conditions, respectively. Dashed lines in (A) and (B) indicate ER/cER at unity. Data are presented as the ER (i.e., mean $\mathrm{P}_{\mathrm{app}}$ from the basolateral to apical side divided by that from the apical to basolateral side) or cER (i.e., ER for MDCKII/hABCG2 cells divided by that for MDCKII/WT cells), obtained from triplicate or quadruplicate runs. 
A

Control

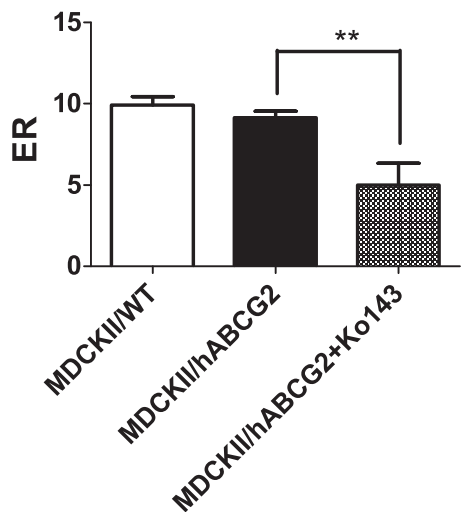

B Verapamil-treated

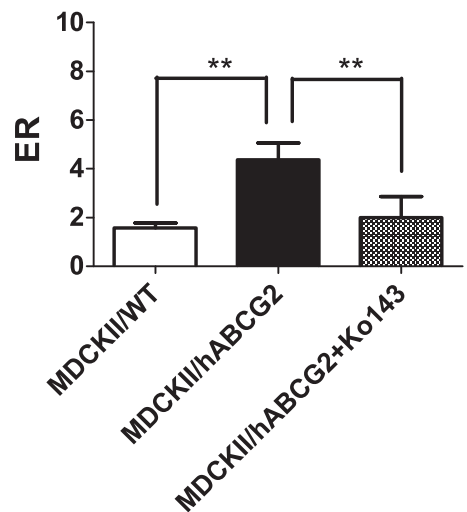

C shRNA-transfected

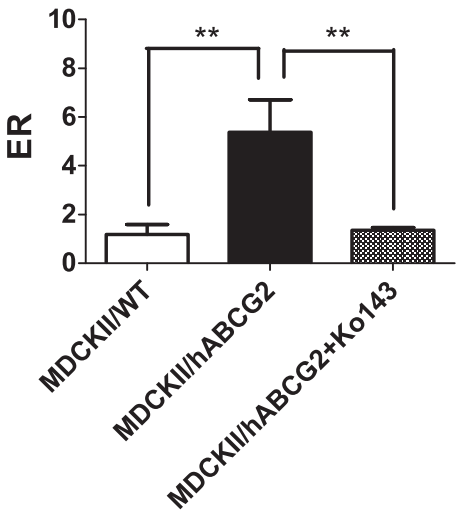

Fig. 4. Bidirectional transport of olaparib in the absence of any suppression (A), in the presence of verapamil treatment (B), or in the presence of shRNAtransfected conditions (C) for MDCKII/WT and MDCKII/hABCG2 cells. When necessary, $1 \mu \mathrm{M}$ Ko143 (indicated as "+Ko143") was added to the incubation mixture. The ER was calculated by dividing the $\mathrm{P}_{\mathrm{app}}$ from the basolateral to apical side by that from the apical to basolateral side. Data are presented as the mean \pm S.D. of three independent experiments, each with quadruplicate runs. Asterisks indicate statistical differences based on oneway analysis of variance, followed by the Tukey post hoc test $(* * P<0.01)$.

hABCG2 (Fig. 1) compared with that in MDCKII/WT cells. As previously noted in the literature, the expression of endogenous transporters can be downregulated as a compensatory response to the transfection and elevated expression of exogenous transporters sharing substrates (Lloyd et al., 1992; Litman et al., 2001; Agarwal et al., 2011). In addition, routine batch-to-batch or passage-dependent differences may manifest alterations in the expression of endogenous transporters (Kuteykin-Teplyakov et al., 2010; Di et al., 2011; Gartzke and Fricker, 2014). Our study aimed to examine the robustness of the cER in relation to hABCG2 and to study experimental methodologies (i.e., kinetic/biochemical suppression) for improving cER estimation accuracy under fluctuating levels of endogenous cABCB1. Thus, the molecular mechanisms underlying the observed downregulation of cABCB1 or any causal relationship between the changes in hABCG2 and cABCB1 were not directly examined. To our knowledge, our study is the first to document that estimating cER can become unreliable for dual substrates of ABCB1 and ABCG2 by the variable expression of cABCB1 in MDCKII

TABLE 2

Bidirectional transport of ABCB1 and/or ABCG2 substrates for MDCKII/WT and MDCKII/hABCG2 cells

\begin{tabular}{|c|c|c|c|c|c|c|}
\hline \multirow{2}{*}{ Inhibitor } & \multirow{2}{*}{ Condition } & \multirow{2}{*}{ Cell Type } & \multicolumn{2}{|c|}{$\mathrm{P}_{\mathrm{app}}$} & \multirow{2}{*}{$\mathrm{ER}$} & \multirow{2}{*}{$\mathrm{cER}$} \\
\hline & & & Apical to Basolateral & Basolateral to Apical & & \\
\hline & & \multicolumn{5}{|c|}{$10^{-6} \mathrm{~cm} / \mathrm{s}$} \\
\hline \multirow[t]{6}{*}{ Digoxin } & No suppression & MDCKII/WT & $1.04 \pm 0.0312$ & $6.53 \pm 0.268$ & 6.3 & 0.7 \\
\hline & & MDCKII/hABCG2 & $1.18 \pm 0.174$ & $5.00 \pm 0.734$ & 4.2 & \\
\hline & Verapamil treatment & MDCKII/WT & $1.55 \pm 0.0764$ & $1.65 \pm 0.0465$ & 1.1 & 1.0 \\
\hline & & MDCKII/hABCG2 & $1.81 \pm 0.0974$ & $1.99 \pm 0.0893$ & 1.1 & \\
\hline & shRNA transfection & MDCKII/WT & $1.32 \pm 0.0286$ & $1.72 \pm 0.0932$ & 1.3 & 0.8 \\
\hline & & MDCKII/hABCG2 & $1.23 \pm 0.290$ & $1.26 \pm 0.167$ & 1.0 & \\
\hline \multirow[t]{6}{*}{ Olaparib } & No suppression & MDCKII/WT & $2.75 \pm 0.414$ & $27.3 \pm 2.72$ & 9.9 & 0.9 \\
\hline & & MDCKII/hABCG2 & $2.73 \pm 0.464$ & $25.1 \pm 4.56$ & 9.2 & \\
\hline & Verapamil treatment & MDCKII/WT & $8.16 \pm 0.164$ & $13.3 \pm 0.514$ & 1.6 & 3.2 \\
\hline & & MDCKII/hABCG2 & $4.40 \pm 0.386$ & $22.5 \pm 1.99$ & 5.1 & \\
\hline & shRNA transfection & MDCKII/WT & $9.35 \pm 0.844$ & $9.83 \pm 0.400$ & 1.1 & 4.9 \\
\hline & & MDCKII/hABCG2 & $4.02 \pm 0.435$ & $20.8 \pm 0.852$ & 5.2 & \\
\hline \multirow[t]{6}{*}{ Irinotecan } & No suppression & MDCKII/WT & $0.611 \pm 0.364$ & $4.47 \pm 0.573$ & 7.3 & 3.0 \\
\hline & & MDCKII/hABCG2 & $0.404 \pm 0.147$ & $8.95 \pm 0.280$ & 22.1 & \\
\hline & Verapamil treatment & MDCKII/WT & $1.60 \pm 0.257$ & $2.34 \pm 0.150$ & 1.5 & 14.3 \\
\hline & & MDCKII/hABCG2 & $0.411 \pm 0.150$ & $8.62 \pm 0.377$ & 21.0 & \\
\hline & shRNA transfection & MDCKII/WT & $1.65 \pm 0.455$ & $1.78 \pm 0.360$ & 1.1 & 13.5 \\
\hline & & MDCKII/hABCG2 & $0.423 \pm 0.045$ & $6.16 \pm 0.263$ & 14.6 & \\
\hline \multirow[t]{6}{*}{ Topotecan } & No suppression & MDCKII/WT & $1.06 \pm 0.0521$ & $4.10 \pm 0.381$ & 3.9 & 5.6 \\
\hline & & MDCKII/hABCG2 & $0.452 \pm 0.0880$ & $9.84 \pm 0.570$ & 21.8 & \\
\hline & Verapamil treatment & MDCKII/WT & $1.67 \pm 0.115$ & $1.86 \pm 0.134$ & 1.1 & 13.1 \\
\hline & & MDCKII/hABCG2 & $0.711 \pm 0.140$ & $10.3 \pm 0.487$ & 14.5 & \\
\hline & shRNA transfection & MDCKII/WT & $1.59 \pm 0.155$ & $1.06 \pm 0.179$ & 0.7 & 13.5 \\
\hline & & MDCKII/hABCG2 & $0.618 \pm 0.221$ & $5.53 \pm 0.449$ & 9.0 & \\
\hline \multirow[t]{6}{*}{ Prazosin } & No suppression & MDCKII/WT & $16.3 \pm 0.527$ & $16.2 \pm 0.683$ & 1.0 & 9.6 \\
\hline & & MDCKII/hABCG2 & $2.76 \pm 0.110$ & $26.1 \pm 1.19$ & 9.5 & \\
\hline & Verapamil treatment & MDCKII/WT & $17.3 \pm 1.91$ & $14.6 \pm 0.880$ & 0.8 & 12.5 \\
\hline & & MDCKII/hABCG2 & $2.66 \pm 0.126$ & $28.0 \pm 1.44$ & 10.5 & \\
\hline & shRNA transfection & MDCKII/WT & $16.3 \pm 0.928$ & $15.0 \pm 0.121$ & 0.9 & 9.9 \\
\hline & & MDCKII/hABCG2 & $3.96 \pm 0.0692$ & $35.8 \pm 1.86$ & 9.1 & \\
\hline
\end{tabular}




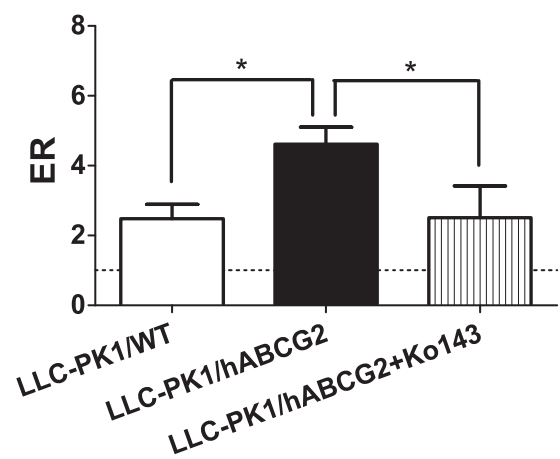

Fig. 5. Bidirectional transport of olaparib in LLC-PK1/WT and LLCPK1/hABCG2 cells. When necessary, $1 \mu \mathrm{M}$ Ko143 (indicated as "+Ko143") was added to the incubation mixture. The ER was calculated by dividing the $\mathrm{P}_{\mathrm{app}}$ from the basolateral to apical side by that from the apical to basolateral side. Data are presented as the mean \pm S.D. of three independent experiments, each with quadruplicate runs. Asterisks indicate statistical differences based on one-way analysis of variance, followed by the Tukey post hoc test $(* P<0.05)$.

cells. In the literature, it was previously noted that the functional expression of cABCB1 was reduced in MDCKII cells after the expression of hABCB1 (Kuteykin-Teplyakov et al., 2010; Li et al., 2013). Taken together, variations in the expression of cABCB1 may not be uncommon in MDCKII cells, especially when foreign transporter genes are introduced into the cell line. In particular, our results, as well as others (Kuteykin-Teplyakov et al., 2010; Li et al., 2013), clearly demonstrate that the variable expression of cABCB1 was associated with an incorrect estimation of cER, leading to inadequate phenotyping of the compounds toward efflux transporters. Considering a number of substrates shared between ABCB1 and ABCG2 (Litman et al., 2001; Agarwal et al., 2011), the possibility of inadequate transporter phenotyping for dual substrates of ABCB1/ABCG2 (e.g., olaparib) cannot be excluded under conditions in which endogenous ABCB1 expression becomes variable. Careful use of the cER in transporter phenotyping is therefore warranted in studies when dual substrates of ABCB1/ABCG2 are being studied in cells expressing these transporters.

In this study, cABCB1 activity was suppressed kinetically or biochemically in an attempt to minimize the contribution of the endogenous efflux system and to improve cER estimation in MDCKII cells. In our kinetic suppression approach, we first screened the selectivity of four well known inhibitors of hABCB1 (zosuquidar, tariquidar, verapamil, and cyclosporine A) against the transport of digoxin and prazosin (which are primarily transported by $\mathrm{cABCB} 1$ and $\mathrm{hABCG} 2$, respectively). We found that verapamil was the most selective toward cABCB1 against hABCG2 (e.g., 191-fold lower $K_{\mathrm{i}}$ value toward cABCB1; Table 1). In parallel, biochemical suppression of cABCB1 expression was also attempted in MDCKII cells using an shRNA approach. In these two separate experimental designs, the basal to apical transport of digoxin was significantly depressed $(P<0.01)$ compared with untreated MDCKII/WT cells (Table 2), with ER values ranging from 1.0 to 1.3 for digoxin in the case of kinetic and biochemical suppression, respectively. These results suggest that these two methods are equally effective in suppressing the contribution of cABCB1. Consistent with this statement, the cER values obtained in the two methods were quite comparable for the three dual substrates (irinotecan, topotecan, and olaparib) of the two efflux transporters (Fig. 3B). Other methods for suppressing cABCB1 function, such as clustered regularly interspaced short palindromic repeats-Cas9-dependent knockout of cABCB1 (Simoff et al., 2016; Karlgren et al., 2017), may also be used to improve the reliability of cER estimation, although the generation and validation of such an experimental system would likely be time-consuming. A selection procedure would not be necessary for the kinetic suppression approach, which would make it immediately applicable.

In a previous study that used an experimental system essentially identical to that used in our study, the investigators reported that hABCG2-mediated efflux was not likely for olaparib since the cER value was calculated to be $0.5-1.0$ (European Medicines Agency, 2014; McCormick and Swaisland, 2016). In the literature, it was also found that an Sf9 membrane vesicle ATPase study was incapable of identifying olaparib as a substrate of hABCG2 (European Medicines Agency, 2014). However, it is noteworthy that the principle aim of the ATPase assay is to estimate transporter activity through the detection of ATP hydrolysis rather than to directly measure the transported substrate itself. Such indirect activity assessment may be more prone to errors, particularly for substrates transported at a reduced rate (namely, weak substrates; Polli et al., 2001; Glavinas et al., 2008; Zhang and Surapaneni, 2012). Interestingly, a distinct decrease was previously noted for basolateral to apical $\mathrm{P}_{\mathrm{app}}$ in the presence of Ko143 (a standard inhibitor for ABCG2) (McCormick and Swaisland, 2016), thus contradicting the conclusion for the cER estimation described in an assessment report from the European Medicines Agency (2014). In our study, the cER of olaparib was $2.81 \pm 0.376$ (kinetic suppression approach, $P<0.01$ ) or $4.62 \pm 0.421$ (biochemical suppression approach, $P<0.01$ ) compared with $0.926 \pm 0.0889$ (without cABCB1 suppression) (Fig. 4), suggesting that hABCG2-mediated efflux cannot be ruled out for olaparib. In addition, the cER was estimated to be close to 2 in hABCG2-overexpressing LLC-PK1 cells, suggesting that hABCG2-mediated transport of olaparib is not limited to one cell system.

Olaparib is an orally active, small molecule inhibitor of poly(ADP-ribose) polymerase. Olaparib was recently approved for the treatment of advanced ovarian cancer and BRCA-mutated metastatic breast cancer, with the possibility of applications to other cancer types as well (Robert et al., 2017). Since olaparib was previously regarded primarily as a substrate for ABCB1, the decrease in intracellular exposure in cancer cells was thought to be entirely mediated by ABCB1 (Vaidyanathan et al., 2016). However, considering the possibility of ABCG2-mediated transport for olaparib, collaborative efflux transport via $\mathrm{ABCB} 1$ and $\mathrm{ABCG} 2$ may be a distinct possibility for the poly(ADP-ribose) polymerase inhibitor in cells or tissues expressing the two transporters. Increased expression of ABCG2 was observed in tumor-bearing mice (Rottenberg et al., 2008) or in a triple-negative breast cancer cell line (Dufour et al., 2015) that had been exposed to olaparib, although the increase was significantly less than that observed for ABCB1. Therefore, further studies are warranted for a complete understanding of the involvement of ABCG2 in the in vivo pharmacokinetics and pharmacodynamics of olaparib.

In conclusion, cABCB1 expression was variable in MDCKII cells expressing hABCG2 compared with untreated MDCKII cells. Both kinetic (the presence of $100 \mu \mathrm{M}$ verapamil) or 
biochemical (cABCB1-targeting shRNA expression) suppression of $\mathrm{cABCB} 1$ appeared to be effective in reducing the contribution of $\mathrm{CABCB} 1$, leading to improved estimation of the cER for dual substrates of ABCB1 and ABCG2 in MDCKII cells. It is particularly noteworthy that the cER for olaparib under conditions in which the function of $\mathrm{CABCB} 1$ was suppressed was consistently greater than 2 in MDCKII/ hABCG2 cells, suggesting that hABCG2-mediated efflux cannot be ruled out for olaparib.

\section{Authorship Contributions}

Participated in research design: Song, Chung.

Conducted experiments: Song, Park, Oh, Hyung, Jeong, Kim.

Performed data analysis: Song, Jeong, Chung.

Wrote or contributed to the writing of the manuscript: Song, Park, Oh, Hyung, Jeong, Kim, Lee, Chung.

\section{References}

Agarwal S, Hartz AM, Elmquist WF, and Bauer B (2011) Breast cancer resistance protein and P-glycoprotein in brain cancer: two gatekeepers team up. Curr Pharm Des 17:2793-2802.

Agarwal S, Sane R, Gallardo JL, Ohlfest JR, and Elmquist WF (2010) Distribution of gefitinib to the brain is limited by P-glycoprotein (ABCB1) and breast cancer resistance protein (ABCG2)-mediated active efflux. J Pharmacol Exp Ther 334:147-155.

Allen JD, van Loevezijn A, Lakhai JM, van der Valk M, van Tellingen O, Reid G, Schellens JH, Koomen GJ, and Schinkel AH (2002) Potent and specific inhibition of the breast cancer resistance protein multidrug transporter in vitro and in mouse intestine by a novel analogue of fumitremorgin C. Mol Cancer Ther 1:417-425.

Cheng Y and Prusoff WH (1973) Relationship between the inhibition constant (K1) and the concentration of inhibitor which causes 50 per cent inhibition (I50) of an enzymatic reaction. Biochem Pharmacol 22:3099-3108.

de Vries NA, Ouwehand M, Buckle T, Beijnen JH, and van Tellingen O (2007) Determination of topotecan in human and mouse plasma and in mouse tissue homogenates by reversed-phase high-performance liquid chromatography. Biomed Chromatogr 21:1191-1200.

Di L, Whitney-Pickett C, Umland JP, Zhang H, Zhang X, Gebhard DF, Lai Y, Federico JJ, 3rd, Davidson RE, Smith R, et al. (2011) Development of a new permeability assay using low-efflux MDCKII cells. J Pharm Sci 100:4974-4985.

Dufour R, Daumar P, Mounetou E, Aubel C, Kwiatkowski F, Abrial C, Vatoux C, Penault-Llorca F, and Bamdad M (2015) BCRP and P-gp relay overexpression in triple negative basal-like breast cancer cell line: a prospective role in resistance to olaparib. Sci Rep 5:12670.

European Medicines Agency (2014) Committee for Medicinal Products for Human Use Assessment Report: Lynparza, European Medicines Agency, London.

Food and Drug Administration (2013) FDA Guidance for Industry: Bioanalytical Method Validation, US Food and Drug Administration Center for Drug Evaluation and Research, Bethesda, MD.

Food and Drug Administration (2017) In Vitro Metabolism and Transporter Mediated Drug-Drug Interaction Studies: Guidance for Industry, US Food and Drug Administration Center for Drug Evaluation and Research, Bethesda, MD.

Gartzke D, Delzer J, Laplanche L, Uchida Y, Hoshi Y, Tachikawa M, Terasaki T, Sydor J, and Fricker G (2015) Genomic knockout of endogenous canine P-glycoprotein in wild-type, human P-glycoprotein and human BCRP transfected MDCKII cell lines by zinc finger nucleases. Pharm Res 32:2060-2071.

Gartzke D and Fricker G (2014) Establishment of optimized MDCK cell lines for reliable efflux transport studies. J Pharm Sci 103:1298-1304.

Glavinas H, Méhn D, Jani M, Oosterhuis B, Herédi-Szabó K, and Krajesi P (2008) Utilization of membrane vesicle preparations to study drug-ABC transporter interactions. Expert Opin Drug Metab Toxicol 4:721-732.

Goh L-B, Spears KJ, Yao D, Ayrton A, Morgan P, Roland Wolf C, and Friedberg T (2002) Endogenous drug transporters in in vitro and in vivo models for the prediction of drug disposition in man. Biochem Pharmacol 64:1569-1578.

Hyung S, Pyeon W, Park JE, Song YK, and Chung SJ (2017) The conditional stimulation of rat organic cation transporter 2 , but not its human ortholog, by mesoridazine: the possibility of the involvement of the high-affinity binding site of the transporter in the stimulation. J Pharm Pharmacol 69:1513-1523.

Ito S, Woodland C, Sarkadi B, Hockmann G, Walker SE, and Koren G (1999) Modeling of P-glycoprotein-involved epithelial drug transport in MDCK cells. Am $J$ Physiol 277:F84-F96.

Jeong Y-S, Yim C-S, Ryu H-M, Noh C-K, Song Y-K, and Chung S-J (2017) Estimation of the minimum permeability coefficient in rats for perfusion-limited tissue distribution in whole-body physiologically-based pharmacokinetics. Eur J Pharm Biopharm 115:1-17.

Karlgren M, Simoff I, Backlund M, Wegler C, Keiser M, Handin N, Müller J, Lundquist P, Jareborg AC, Oswald S, et al. (2017) A CRISPR-Ccas9 generated MDCK cell line expressing human MDR1 without endogenous canine MDR1 (cABCB1): an improved tool for drug efflux studies. J Pharm Sci 106:2909-2913.

Kuteykin-Teplyakov K, Luna-Tortós C, Ambroziak K, and Löscher W (2010) Differences in the expression of endogenous efflux transporters in MDR1-transfected versus wildtype cell lines affect P-glycoprotein mediated drug transport. $\mathrm{Br} J$ Pharmacol 160:1453-1463.

Laemmli UK (1970) Cleavage of structural proteins during the assembly of the head of bacteriophage T4. Nature 227:680-685.
Lee JH, Noh CK, Yim CS, Jeong YS, Ahn SH, Lee W, Kim DD, and Chung SJ (2015) Kinetics of the absorption, distribution, metabolism, and excretion of lobeglitazone, a novel activator of peroxisome proliferator-activated receptor gamma in rats. $J$ Pharm Sci 104:3049-3059.

Lepist E-I, Phan TK, Roy A, Tong L, Maclennan K, Murray B, and Ray AS (2012) Cobicistat boosts the intestinal absorption of transport substrates, including HIV protease inhibitors and GS-7340, in vitro. Antimicrob Agents Chemother 56:5409-5413.

Li J, Wang Y, and Hidalgo IJ (2013) Kinetic analysis of human and canine P-glycoprotein-mediated drug transport in MDR1-MDCK cell model: approaches to reduce false-negative substrate classification. J Pharm Sci 102:3436-3446.

Litman T, Druley TE, Stein WD, and Bates SE (2001) From MDR to MXR: new understanding of multidrug resistance systems, their properties and clinical significance. Cell Mol Life Sci 58:931-959.

Lloyd C, Schevzov G, and Gunning P (1992) Transfection of nonmuscle beta- and gamma-actin genes into myoblasts elicits different feedback regulatory responses from endogenous actin genes. J Cell Biol 117:787-797.

Luo FR, Paranjpe PV, Guo A, Rubin E, and Sinko P (2002) Intestinal transport of irinotecan in Caco-2 cells and MDCK II cells overexpressing efflux transporters Pgp, cMOAT, and MRP1. Drug Metab Dispos 30:763-770.

McCormick A and Swaisland H (2016) In vitro assessment of the roles of drug transporters in the disposition and drug-drug interaction potential of olaparib. Xenobiotica 47:903-915.

Polli JW, Wring SA, Humphreys JE, Huang L, Morgan JB, Webster LO, and SerabjitSingh CS (2001) Rational use of in vitro P-glycoprotein assays in drug discovery. $J$ Pharmacol Exp Ther 299:620-628.

Robert M, Frenel J-S, Gourmelon C, Patsouris A, Augereau P, and Campone M (2017) Olaparib for the treatment of breast cancer. Expert Opin Investig Drugs 26:751-759.

Römermann K, Helmer R, and Löscher W (2015) The antiepileptic drug lamotrigine is a substrate of mouse and human breast cancer resistance protein (ABCG2). Neuropharmacology 93:7-14.

Rottenberg S, Jaspers JE, Kersbergen A, van der Burg E, Nygren AO, Zander SA, Derksen PW, de Bruin M, Zevenhoven J, Lau A, et al. (2008) High sensitivity of BRCA1-deficient mammary tumors to the PARP inhibitor AZD2281 alone and in combination with platinum drugs. Proc Natl Acad Sci USA 105:17079-17084.

Scharenberg CW, Harkey MA, and Torok-Storb B (2002) The ABCG2 transporter is an efficient Hoechst 33342 efflux pump and is preferentially expressed by immature human hematopoietic progenitors. Blood 99:507-512.

Scheffer GL, Kool M, Heijn M, de Haas M, Pijnenborg AC, Wijnholds J, van Helvoort A de Jong MC, Hooijberg JH, Mol CA, et al. (2000) Specific detection of multidrug resistance proteins MRP1, MRP2, MRP3, MRP5, and MDR3 P-glycoprotein with a panel of monoclonal antibodies. Cancer Res 60:5269-5277.

Schinkel AH, Roelofs EM, and Borst P (1991) Characterization of the human MDR3 $\mathrm{P}$-glycoprotein and its recognition by $\mathrm{P}$-glycoprotein-specific monoclonal antibodies. Cancer Res 51:2628-2635.

Siissalo S, Laitinen L, Koljonen M, Vellonen K-S, Kortejärvi H, Urtti A, Hirvonen J, and Kaukonen AM (2007) Effect of cell differentiation and passage number on the expression of efflux proteins in wild type and vinblastine-induced Caco-2 cell lines. Eur J Pharm Biopharm 67:548-554

Simoff I, Karlgren M, Backlund M, Lindström AC, Gaugaz FZ, Matsson P, and Artursson P (2016) Complete knockout of endogenous Mdr1 (Abcb1) in MDCK cells by CRISPR-Cas9. J Pharm Sci 105:1017-1021.

Smith PK, Krohn RI, Hermanson GT, Mallia AK, Gartner FH, Provenzano MD, Fujimoto EK, Goeke NM, Olson BJ, and Klenk DC (1985) Measurement of protein using bicinchoninic acid. Anal Biochem 150:76-85.

Sparidans RW, Martens I, Valkenburg-van Iersel LB, den Hartigh J, Schellens JH, and Beijnen JH (2011) Liquid chromatography-tandem mass spectrometric assay for the PARP-1 inhibitor olaparib in combination with the nitrogen mustard melphalan in human plasma. J Chromatogr B Analyt Technol Biomed Life Sci 879:1851-1856.

Stephens RH, O’Neill CA, Warhurst A, Carlson GL, Rowland M, and Warhurst G (2001) Kinetic profiling of P-glycoprotein-mediated drug efflux in rat and human intestinal epithelia. J Pharmacol Exp Ther 296:584-591.

Tang F, Horie K, and Borchardt RT (2002) Are MDCK cells transfected with the human MDR1 gene a good model of the human intestinal mucosa? Pharm Res 19:765-772. Taub ME, Kristensen L, and Frokjaer S (2002) Optimized conditions for MDCK permeability and turbidimetric solubility studies using compounds representative of BCS classes I-IV. Eur J Pharm Sci 15:331-340.

Taub ME, Podila L, Ely D, and Almeida I (2005) Functional assessment of multiple P-glycoprotein (P-gp) probe substrates: influence of cell line and modulator concentration on P-gp activity. Drug Metab Dispos 33:1679-1687.

Vaidyanathan A, Sawers L, Gannon A-L, Chakravarty P, Scott AL, Bray SE, Ferguson MJ, and Smith G (2016) ABCB1 (MDR1) induction defines a common resistance mechanism in paclitaxel- and olaparib-resistant ovarian cancer cells. Br J Cancer 115:431-441.

Wang Q, Rager JD, Weinstein K, Kardos PS, Dobson GL, Li J, and Hidalgo IJ (2005) Evaluation of the MDR-MDCK cell line as a permeability screen for the blood-brain barrier. Int J Pharm 288:349-359.

Xia CQ, Liu N, Yang D, Miwa G, and Gan LS (2005) Expression, localization, and functional characteristics of breast cancer resistance protein in Caco-2 cells. Drug Metab Dispos 33:637-643.

Yim CS, Jeong YS, Lee SY, Pyeon W, Ryu HM, Lee JH, Lee KR, Maeng HJ, and Chung SJ (2017) Specific inhibition of the distribution of lobeglitazone to the liver by atorvastatin in rats: evidence for a rat organic anion transporting polypeptide 1B2mediated interaction in hepatic transport. Drug Metab Dispos 45:246-259.

Zhang D and Surapaneni S (2012) ADME-enabling technologies in drug design and development. John Wiley \& Sons.

Address correspondence to: Dr. Suk-Jae Chung, College of Pharmacy, Seoul National University, 1 Gwanak-ro, Gwanak-gu, Seoul 08826, Republic of Korea. E-mail: sukjae@snu.ac.kr 